\title{
El psicólogo educacional en contextos educativos no formales e informales
}

\author{
The educational psychologist in non-formal and informal \\ educational settings
}

\author{
Luis Alberto Rodríguez de los Ríos' \\ Universidad Nacional de Educación Enrique Guzmán y Valle \\ rodri0351@hotmail.com
}

\section{RESUMEN}

La educación es un fenómeno complejo que abarca desde los aspectos puramente cuantitativos y tangibles hasta los aspectos cualitativos e ideológicos, que incluye una gran cantidad de variables $y$ múltiples aspectos que se manifiestan en el mismo hecho educativo. Una propuesta de tipología de la educación, identifica a la educación formal, no formal e informal, como respuesta alternativa a las críticas que dos décadas antes habían planteado mentores de las teorías críticas de la educación sobre la institución escolar, quienes valoraban otros entornos educativos en expansión y hasta alguno de ellos proponía la desescolarización de la sociedad, aceptándose así que la educación institucionalizada es solo una parte de lo educativo. La psicología educacional, por su parte, viene desarrollando nuevas tendencias; en el ámbito conceptual, se observa un consenso en cambiar la definición convencional del psicólogo educacional por otra; así el psicólogo de la educación es el profesional de la psicología cuyo objetivo de trabajo es la reflexión e intervención sobre el comportamiento humano, en situaciones educativas, mediante el desarrollo de la capacidad de las personas, grupos e instituciones. Se entiende en término educativo el sentido más amplio de formación y desarrollo personal y colectivo. El psicólogo de la educación desarrolla su actividad profesional principalmente en el marco de los sistemas sociales dedicados a la educación en todos sus diversos niveles y modalidades.

Palabras clave: Psicologo educacional, educación formal, educación no formal, educación informal.

\begin{abstract}
Education is a complex phenomenon that ranges from purely quantitative and qualitative aspects to tangible and ideological ones, including a large variable number and multiple forms that manifest themselves in the same educational event. A proposed education typology identifies the formal, non-formal and informal education, as an alternative response to criticism raised two decades earlier by education mentors on critical theories referred to the school, who valued other educational environments' expansion and even some of them proposed the deschooling society and accepted that formal education is only a part of education. Educational psychology, meanwhile, has developed new directions; on the conceptual level, consensus is seen to change the conventional definition of educational psychologist for another; thus, educational psychologist is the professional whose work involves objective reflection and intervention on human behavior in educational situations, through the development of the individuals' capacity, groups and institutions. The educational psychologist primarily develops his working profession in the context of social systems dedicated to education in all levels and modalities.
\end{abstract}

Keywords: Educational psychologist, formal, nonformal education, informal education.

Historial del artículo:

Recibido: 27 de mayo de 2015. Aprobado: 13 de agosto de 2015. Disponible en línea: 30 de diciembre de 2015

1 Doctor en Psicología y en Educación. Director de la Escuela de Posgrado de la Universidad Nacional de Educación Enrique Guzmán y Valle (Lima-Perú). 


\section{INTRODUCCIÓN}

Una de las características de la sociedad contemporánea es el cambio múltiple, acelerado y constante que se experimenta en todos los ámbitos de la actividad humana merced a un conjunto de transformaciones ocurridas desde hace más de tres décadas en el campo de la ciencia, la tecnología (informática, telecomunicaciones, robótica, etc.) y la economía (globalización).

Paraalgunosanalistas, el cambiosocietal experimentado ha configurado un contexto sociocultural denominado sociedad de la información, del conocimiento y/o del permanente aprendizaje, o también era de la turbulencia, la incertidumbre y del constante riesgo, expresado básicamente en dos fenómenos evidentes: el rol privilegiado de la información y el conocimiento en la actividad económica, y la relatividad de las normas sociales, culturales, políticas, ideológicas y axiológicas. Todo ello ha impactado significativamente en los procesos educativos, expresado en un conjunto de retos y desafíos en la tarea de formar a las futuras generaciones.

En el marco de una sociedad compleja y la necesidad de cambios educativos, la psicología educativa se ha constituido en los últimos tiempos en uno de los referentes disciplinares más importantes para la implementación de las innovaciones psicopedagógicas y para el afrontamiento de los principales problemas formativos que manifiestan los estudiantes del presente milenio.

\section{Conceptualizaciones sobre educación}

Desde la antigüedad hasta hoy se han ensayado y propuesto diversas conceptualizaciones sobre educación, algunas coincidentes, otras divergentes. Probablemente, las divergencias tengan su origen en la ambivalencia etimológica (1), pues educación proviene de las voces latinas educere, que significa "hacer salir", y educare, que alude a "criar", "alimentar" o "producir". Pese a las diferentes posturas para definir la educación, se observan criterios comunes en ellas, al entenderla como un proceso intencional y sistemático relacionado con el perfeccionamiento individual y social del ser humano en función de un planteamiento axiológico o ideológico determinado.

Una de las conceptualizaciones más completas sobre educación, a nuestro entender, es la enunciada por Guédez, quien la concibe como un "fenómeno complejo que abarca desde los aspectos puramente cuantitativos y tangibles hasta los aspectos cualitativos e ideológicos, que incluye una gran cantidad de variables y múltiples aspectos que se manifiestan en el mismo hecho educativo" (2).
Afirma que la educación es fundamentalmente un fenómeno social, histórico e ideológico. "Es de origen social porque nace en la sociedad y con la sociedad, es de naturaleza social, porque se administra a través de las distintas instituciones que conforman la realidad social —llámese escuelas, colegios, institutos, programas no escolarizados o influencia espontánea-, y es de finalidad social, porque la sociedad basa su propia expectativa de supervivencia en la educación misma" (2). Sin embargo, la educación no es solo un fenómeno de naturaleza social, sino también un fenómeno biológico, toda vez que el ser humano está dotado de un sistema nervioso altamente desarrollado, como el cerebro, que le permite gestionar su capacidad de educación.

Además, la educación implica un aspecto psicológico que se expresa en la existencia de una actividad mental que se define por una capacidad intelectual, afectiva y volitiva que le hace susceptible de la educatividad y de la educabilidad previstas en el propio proceso educativo. A ello se suma la variable de carácter lingüístico, en la medida que el lenguaje -especialmente el verbal- constituye una herramienta fundamental de comunicación y de expresión y una parte del proceso educativo recae y se apoya básicamente sobre esa capacidad comunicativa del hombre.

La dimensión histórica de la educación está ligada a la variable antropológica, "porque siempre la educación representará una posibilidad de transmisión de la herencia cultural del patrimonio histórico de una generación a otra" (2). Ello supone que la educación como proceso ocurre en un espacio y un tiempo determinado que evoluciona en función de los cambios que se operan en las culturas a través de las distintas épocas y en determinados contextos socio-culturales, económicos, geográficos y políticos. Al respecto, Guédez sostiene que "puede entenderse la educación como un proceso que se mueve con historia y que, al mismo tiempo, mueve a la historia. La educación se mueve con la historia porque ella recibe y expresa los cambios estructurales que se operan en una determinada realidad social" (2).

Esta dimensión permite entrever la dimensión ideológica de la educación, pues, como todo proceso social e histórico, conlleva todo un esquema ideológico signalizado por un conjunto de aspiraciones, apetencias, deseos y necesidades e intereses sociales marcados por una elección. "Ellas en su conjunto van a desplazarse hacia sus conquistas y consolidaciones. Los proyectos pedagógicos buscan en la educación el recurso o vehículo para facilitar sus logros y robustecer sus prospectivas. Es así cómo se establecen las relaciones entre ideología y educación" (2). 


\section{Los escenarios educativos}

En 1985, Combs (3) propone una tipología de la educación, identifica a la educación formal, no formal e informal, como respuesta alternativa a las críticas que dos décadas antes habían planteado mentores de las teorías críticas de la educación sobre la institución escolar $(4,5,6,7)$, quienes valoraban otros entornos educativos en expansión y hasta alguno de ellos proponía la desescolarización de la sociedad, aceptándose así que la educación institucionalizada es solo una parte de lo educativo.

La educación formal está representada por la educación institucionalizada, constituida por todo un sistema jerárquicamente organizado -niveles, modalidades, programas, etc.-, cronológicamente graduado y oficialmente aceptado con fines de certificación, promoción y acreditación académica, técnica y/o profesional.

La educación no formal se define como "cualquier actividad educativa organizada, sistemática, impartida fuera del marco del sistema formal, para suministrar determinados tipos de aprendizaje a subgrupos concretos de la población, tanto adultos como niños" (3).

La educación informal hace referencia al proceso en el transcurso de toda la vida a través del cual cada individuo adquiere actitudes, valores, destrezas y conocimientos de la experiencia diaria y de las influencias y recursos educativos de su entorno -de la familia y niños, del trabajo y el juego, en el mercado, la biblioteca y en los medios de comunicación, incluyendo las nuevas tecnologías-.

La educación formal y no formal son intencionales, mientras que la informal no lo es. La formal se identifica con lo institucional y normado y la no formal con la no institucional y apartada de sus normas. Tirado, et al. (8), desde la perspectiva de la psicología educativa, prefieren hablar de escenarios educativos, al referirse a los tipos de educación; así identifican a la educación familiar como escenario de la psicología I, a la educación institucional como escenario de la psicología educativa II y a las instituciones educativas y ambientes culturales formativas como escenarios de la psicología educativa III, ubicando a la educación no formal e informal en este último escenario y aludiéndolas como instituciones coeducativas.

Echeverria (8) considera tres tipos de escenarios educativos generales en el desarrollo de la humanidad, el entorno natural, el urbano y el ciberespacio.

El primer escenario, el natural, es nuestro entorno inmediato, representado por la familia -o la tribu-, y constituye el primer agente educativo de socialización y de apropiación de las herramientas culturales básicas de subsistencia, de adaptación social y productiva; utiliza como medio comunicativo la lengua materna, la tradición oral para mantener la memoria del grupo, así como los juegos y fiestas locales para la diversión $y$ el entretenimiento.

El segundo escenario es el urbano, que se desarrolla con la aparición de las ciudades y el Estado, en el cual surge la escuela como agente educativo fundamental, cuya función es ampliar los procesos educativos del primer escenario e introducir otros nuevos, relacionados con la alfabetización básica -lectura, escritura, aritmética-, así como un conjunto de saberes conceptuales, para adaptarse socialmente en función de normas y códigos simbólicos de convivencia en los diferentes escenarios urbanos e insertarse al aparato productivo eficazmente. En este escenario, la escritura es el nuevo gran instrumento educativo, a través de la cual se potencia la capacidad de comunicación, de memorización, transmisión de información y construcción de saberes disciplinares.

Por la década de 1960, aparece en este escenario urbano la educación no formal, que consiste en trasladar al niño a escenarios especialmente diseñados para desarrollar en ellos procesos educativos: aulas, patios de colegio, bibliotecas, museos, parques zoológicos, etc., con lo que se introduce otros agentes educativos diferentes a la familia, la escuela y el Estado, como las asociaciones, por ejemplo, las organizaciones no gubernamentales (ONG) y la comunidad (8).

El tercer escenario corresponde al ciberespacio, desarrollado por las tecnologías de comunicación, telecomunicación en información, las cuales se encuentran omnipresentes en la vida cotidiana urbana, desde los domésticos hasta los organizacionales productivos, pasando por toda la gama de interacciones sociales y recreativas. Comporta nuevas maneras de trabajar, de comunicarnos, relacionarnos, aprender, pensar y ver el mundo, configurando una nueva realidad: la realidad virtual.

En este entorno las personas aprenden de manera informal -televisión, videojuegos, internet, etc.., constituyendo un nuevo espacio educativo -educación sin paredes-, generando diversos escenarios -comunidades de aprendizaje-, posibilidades y retos que el psicólogo educacional debe considerar, en especial, por su impacto en las sociedades y personas (8).

Vale destacar que los tres tipos de educación o escenarios no deben ser entendidos como entidades separadas sino como modalidades predominantes de aprendizaje, las cuales pueden coexistir, algunas veces articuladas y otras desarticuladas, porque en el transcurso de la vida aprendemos con las tres formas 
de educación.

\section{Nuevas tendencias de la psicología educativa}

Lo descrito tiene el propósito de destacar los nuevos escenarios educativos desarrollados socialmente en las últimas décadas y aceptadas formalmente por las ciencias de la educación, en especial, por la pedagogía (9).

La psicología educacional, por su parte, viene desarrollando nuevas tendencias tanto en su aspecto conceptual como de intervención especializada. En el ámbito conceptual, se observa un consenso en cambiar la definición convencional del psicólogo educacional por otra más de acuerdo con los nuevos escenarios y
Desde el punto de intervención psicoeducativa, la función del psicólogo educacional, se ha ampliado hacia espacios e instituciones socioeducativas no formales e informales, diferentes al ámbito escolar.

Todas ellas en función de la presencia y omnipresencia de las nuevas tecnologías de información y comunicación (TIC) y a las nuevas concepciones educativas, desarrolladas contemporáneamente, como la educación permanente, la educación para todos, aprendizaje virtual, educación a lo largo de la vida, educación alternativa, aprendizaje situado, entre otras.

Estas nuevas funciones están marcando una nueva tendencia de actuación académica y profesional del psicólogo educacional desde una perspectiva multi e interdisciplinaria, pues los escenarios educativos no

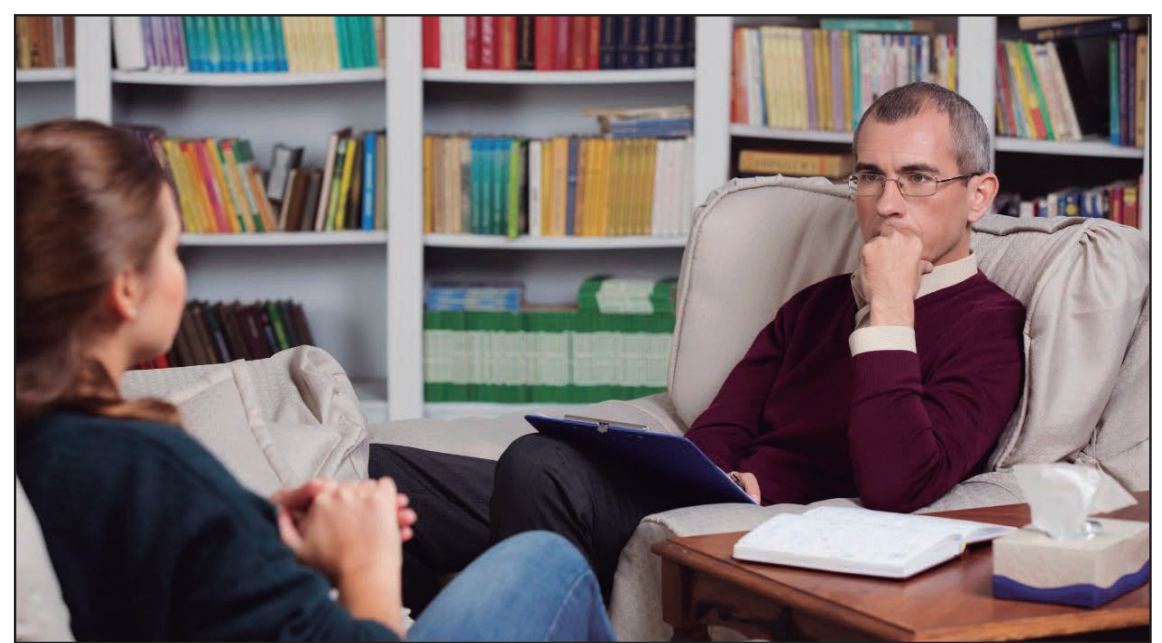

Figura $N^{\circ}$ 1: El psicólogo educacional cumple funciones de capacitación y formación dirigidas a agentes educativos como padres, docentes, directivos.

Fuente: http://saludhoy.org/tag/psicologo/

los retos educativos en el presente milenio.

En este sentido, la definición del psicólogo de la educación que propone el Colegio Oficial de Psicólogos de España (1998) es coherente con esta tendencia, cuando afirma: "El psicólogo de la educación es el profesional de la psicología cuyo objetivo de trabajo es la reflexión e intervención sobre el comportamiento humano, en situaciones educativas, mediante el desarrollo de la capacidad de las personas, grupos e instituciones. Se entiende en término educativo el sentido más amplio de formación y desarrollo personal y colectivo. El psicólogo de la educación desarrolla su actividad profesional principalmente en el marco de los sistemas sociales dedicados a la educación en todos sus diversos niveles y modalidades; tanto en los sistemas reglados, no reglados, formales e informales y durante todo el ciclo vital de la persona" (10). formales e informales exigen un trabajo compartido de distintos profesionales, vale decir, un trabajo en equipo, buscando la integración de las actividades y resultados a partir de una división funcional del trabajo.

Es importante anotar, por otro lado, que la intervención psicoeducativa en las modalidades o escenarios educativos descritos se basan en el diseño, ejecución y evaluación de programas que pretenden potenciar, mejorar o solucionar situaciones socioeducativas, según sea el caso, de naturaleza compleja y multidimensionales, obligando a contemplar distintos niveles de actuación; individualizado, grupal o comunitario.

\section{El psicólogo educacional en el nuevo contexto}

Hoy el psicólogo educacional debe asumir su labor 
desde la perspectiva del desarrollo humano integral y desde un enfoque holístico del proceso educativo. Para ello debe ser consciente que su práctica profesional no se reduce al ámbito educacional institucional, sino que traspasa sus muros hacia una práctica social o socioeducativa que tiene que ver más con los aprendizajes implícitos -adaptivos o desadaptativos y sus respectivas potencialidades- que con los explícitos o formales. "En este sentido -como destacan Castro, Domínguez y Sánchez-, el psicólogo educativo debe acompañar, investigar y comprender actividades que le permitan colaborar y apoyar educativamente a individuos, grupos y comunidades con los problemas e inquietudes relevantes en su desarrollo como sujetos sociales coadyuvando a la mejora de la calidad de vida y bienestar" (11).

Esta práctica social la concreta en la realización de acciones como diseñador $\mathrm{u}$ orientador instruccional, de promoción, prevención, intervención e investigación en contextos educativos no formales e informales en la comunidad de pertenencia o de referencia impulsados y desarrollados por los entes gubernamentales -gobiernos: central, regional, provincial o distrital-, mediante sus políticas de acción social o acción socioeducativa o de aquellas desarrolladas por las propias organizaciones sociales de base.

Las funciones de diseñador $u$ orientador instruccional son transversales y permanentes, pues debe cumplirlas en todas las demás que son de su competencia, especialmente en la planificación, ejecución y evaluación de programas socioeducativos, de salud, seguridad ciudadana, formación de ciudadanía, prevención de riesgos de todo tipo, en particular los de naturaleza psicosocial, y poniendo énfasis en los aspectos psicológicos de la instrucción, el diseño de la instrucción y las respectivas estrategias cualitativas.

Las funciones de promoción están dirigidas más al desarrollo de las potencialidades o virtualidades de los individuos o grupos sociales, a través de la estimulación y fortalecimiento de las habilidades cognitivas - pensamiento crítico, pensamiento creativo, toma de decisiones y resolución de problemas, entre otras-, el desarrollo de habilidades socioecomocionales -manejo de emociones- y prosociales, la capacidad de afrontamiento y resiliencia, el aprendizaje eficaz y autónomo, en especial las habilidades y estrategias relacionadas con el aprendizaje virtual y la navegación en el ciberespacio; así como las fortalezas humanas en el marco de la psicología positiva.

El psicólogo educacional también cumple tareas de asesoría, consejería en el desarrollo y elaboración de materiales educativos -convencionales y/o digitalesen la capacitación del personal responsable de las acciones específicas, en función de modelos teóricos instruccionales validados empíricamente, con el propósito de lograr adecuados niveles de eficacia, eficiencia y sostenibilidad.

Las funciones de prevención estarían dirigidas, especialmente, a la prevención primaria de trastornos psicosociales, a las adicciones de todo tipo, en particular las emergentes -endopatías, a las compras, al tabaco, al sexo, el ejercicio físico y a las tecnologías, video juegos, internet, celulares y las dependencias sentimentales-; a todo tipo de violencia -psicológica, física, sexual, etc.-, desajustes familiares, conflictos económicos, sociales y comunitarios; en pro de una convivencia social armoniosa, de la construcción de una coyuntura de paz y de prevención en el transcurso del ciclo vital.

En la "sociedad de riesgo" en la que vivimos, el objetivo es reducirlos a lo mínimo posible, objetivo que es compartido por las ciencias sociales y de la salud, donde la psicología cumple un papel relevante, en especial la psicología de la educación, teniendo en cuenta que tanto las conductas positivas o adaptativas como las negativas, desadaptativas o de riesgo, suponen procesos de aprendizaje experienciales, cotidianos e implícitos, mayoritariamente.

Considerando que la función preventiva, así como la de promoción, implican fundamentalmente acciones educativas, persuasivas y motivacionales, bien podemos enmarcar estas funciones del psicólogo educacional en el movimiento o políticas públicas de educación para la salud, en particular de la educación psicosocial, tan descuidado en los últimos tiempos por las políticas gubernamentales e institucionales especializadas a nivel local, regional y nacional. Hoy, más que nunca, se hace necesario capacitar a la población -familia, niñez y adolescencia-, prioritariamente para que aumente el control de su propia salud y la mejore, según recomienda la OMS (12), promoviendo el conocimiento y la adopción de conductas saludables, orientadas a lograr una mejor calidad de vida y bienestar posibles.

Las funciones de intervención comprenden el desarrollo de acciones específicas, a partir de dos componentes, el tecnológico-proyectivo y el técnico-práctico. El primero se refiere al conocimiento tecnológico relacionado con el desarrollo de modelos y propuestas educativas generales para la intervención, y el segundo, al conjunto de recursos técnicos específicos para la intervención y solución de problemas en situaciones educativas concretas, así como para el ejercicio concreto de la práctica profesional (8).

Desde la perspectiva de la educación no formal e informal, el componente tecnológico-proyectivo de la intervención psicoeducativa es todo un espacio que muestra un desarrollo incipiente, en especial todo lo relacionado con la influencia de las $\mathrm{TIC}$ a los procesos educativos y aprendizajes en contextos informales o 
escenarios socioculturales potencialmente educativos y socioformativos, situación que está ligada a procesos sistemáticos de reflexión e investigación empírica rigurosa, constituyendo una tarea pendiente en cuanto a propuestas y modelos innovadores para la intervención psicoeducativa.

En la actual sociedad del conocimiento el psicólogo educacional tiene la necesidad de desarrollar competencias profesionales que le permitan aplicar estrategias desde el enfoque de aprendizaje estratégico, el cual hace posible que gestione sus propios conocimientos, adopte una autonomía creciente en sus actividades académicas, laborales, recreativas, disponga de herramientas sociales e intelectuales que faciliten un aprendizaje continuo en el transcurso de la vida, promoviendo el aprendizaje colaborativo, la educación en valores y el manejo de nuevas tecnologías de información y comunicación.

Según Tirado et al., el psicólogo educacional centra sus intervenciones "en la promoción del desarrollo de la personalidad a lo largo del ciclo de la vida, para lograr su realización personal y académica, o su inclusión social y bienestar emocional" (8), así como la mejora de los escenarios educativos. Estas intervenciones, actualmente, se han extendido a escenarios educativos no escolarizados muy diversos como comunidades, empresas, etc., buscando empoderamiento de las personas en cuanto a la autogestión y el autoaprendizaje.

Entre las estrategias de intervención psicoeducativa más relevantes en los contextos educativos no formales e informales tenemos:

\section{Entrenamiento y formación de agentes educativos}

El psicólogo educacional cumple funciones de capacitación y formación dirigidas a agentes educativos como padres, docentes, directivos, pares, animadores sociales, entrenadores deportivos, promotores de salud, etc. Su preparación profesional lo habilitan para realizar diagnósticos contextualizados de necesidades de capacitación en los escenarios de la educación no formal e informal y sobre esa base diseñar, ejecutar y evaluar los programas pertinentes, desde las modalidades: presencial, semipresencial y/o virtual.

\section{Consultoría, orientación y tutoría psicoeducativa}

Realiza acciones profesionales de consultor o asesor en aspectos especializados relacionados con el aprendizaje, la enseñanza y el desarrollo humano, en especial en centros de servicios sociales comunitarios, centros de atención a la mujer, centros de atención al niño, centros de atención a la juventud, centros de atención al drogodependiente, centros de entrenamiento ocupacional, entre otros. Asimismo, desarrolla labores de orientación y tutoría académica, con el propósito de favorecer el logro de aprendizajes eficaces de los tutoriados, mediante acciones de fortalecimiento de capacidades académicas y personales para la adecuada toma de decisiones, solución de conflictos y la construcción de planes y proyectos de vida.

\section{Atención a poblaciones en situaciones de exclusión y riesgo}

Su competencia profesional le permite actuar en el desarrollo de políticas, programas y estrategias dirigidas a las poblaciones, vulnerables y en situaciones de exclusión. En las últimas décadas los gobiernos de la región, así como la sociedad civil y diversas organizaciones están implementando acciones inclusivas socioeducativas dirigidas a niños, adolescentes y adultos con necesidades educativas especiales, en situación de calle, víctimas de violencia política, migrantes, indígenas, maltratados, entre otras. La labor de este profesional se centra básicamente en el empoderamiento personal y social de dichas poblaciones -fortalecer la identidad y la preparación para la vida en sociedad-, mediante la búsqueda de oportunidades, la eliminación de barreras para participar en todo los ámbitos de la vida, el énfasis en la equidad y la participación amplia de esta población en la sociedad (8).

En general, las funciones del psicólogo educacional tienden a la promoción del desarrollo personal, cognitivo, académico, emocional y social, participando a nivel multi e interdisciplinaria y/o especializada.

En cuanto a la función de investigación, tiene un fecundo espacio de situaciones problemáticas a estudiar. Hay un vacío de conocimientos acerca del impacto de los cambios locales, políticos, tecnológicos y culturales en contextos de la educación no formal e informal -verbigracia, en los aprendizajes implícitos, espontáneos y cotidianos-; sobre la validación de modelos y estrategias para el logro de procesos educativos de calidad en entornos de pobreza, marginación y exclusión social; sobre estrategias de formación complementaria del niño y adolescentes, de padres y madres que sobretrabajan y no tienen tiempo disponible para atender sus necesidades de aprendizaje -no solamente académicas, sino también socioafectivas, axiológicas y morales-: sobre la evaluación de los sistemas, programas y estrategias de enseñanza online o a distancia dirigidos a poblaciones -jóvenes y adultos- excluidos del sistema escolar formal -por ejemplo, cursos técnicos, de calificación ocupacional, etc.-; sobre el aprovechamiento educativo de las redes sociales, internet y en general, 
de los entornos digitales, así como su influencia en los procesos de desarrollo y socialización.

\section{CONCLUSIONES}

Es pertinente la ejecución de trabajos sobre la efectividad y confiabilidad de programas de intervención en conductas de riesgo o adicción -convencionales y emergentes - y preventivos relacionados con la sexualidad en todas sus dimensiones e implicancias. Asimismo, sobre estrategias psicoeducativas para el desarrollo de escenarios culturales en ambientes naturales con potencialidades educativas.

La literatura a investigar es amplia, diversa, multidimensional, pluriparadigmática y multimetodológica, todo lo cual conlleva que el psicólogo educacional, además de trabajar en equipo, esté premunido de herramientas conceptuales y metodológicas provenientes del enfoque cuantitativo -descripción y explicación- y del enfoque cualitativo -interpretación y comprensión- para abordar con disposición holística los procesos y prácticas educativas que le dan crédito en los mencionados escenarios, así como las problemáticas sociales a nivel individual, grupal y comunitario, privilegiando la estrategia metodológica de la investigación-acción.

\section{REFERENCIAS BIBLIOGRÁFICAS}

1. Colom AJ, Bernabeu JW, Dominguez E \& Sarramona UJ. Teorías e instituciones contemporáneas de la educación. Barcelona: Ariel; 2001.
2. Guédez V. La formulación de un modelo académico curricular como referencia para las definiciones de las alternativas de la educación superior en la década de los 80. La Educación. 1983; 27(92): 67-86.

3. Coombs P. La crisis mundial de la educación. Madrid: Santillana; 1985.

4. Carpenter E \& McLuhan M. El aula sin muros. Investigaciones sobre técnicas de comunicación. 2a ed. Barcelona: LAIA; 1974.

5. Freire P. La educación como práctica de la libertad. Buenos Aires: Siglo XXI; 1979.

6. Goodman P. La deseducación obligatoria. Barcelona: Fontanella; 1973.

7. Illich I. La sociedad desescolarizada. Barcelona: Barral; 1974.

8. Tirado $F$, Martínez $M$, Covarrubias $P$, López $M$, Quesada R, Olmos A, et al. Psicología educativa para afrontar los desafíos del siglo XXI. México: McGraw-Hill; 2011.

9. UNESCO. Terminología: Tesauro de la educación. París: UNESCO; 1977.

10. Colegio Oficial de Psicólogos de España. Psicología de la educación. En Perfiles profesionales del psicólogo. España; 1998. Recuperado de http:// www.cop.es/perfiles/contenido/educacion.pdf

11. Gravini M, Porto A \& Escorcia L. El psicólogo educativo en la actualidad: un facilitador del desarrollo humano integral. Psicogente. 2010; 13(23): 158-163.

12. Oblitas L. Psicología de la salud y calidad de vida. México: Thomson; 2004. 